\title{
Direct numerical simulation of transition and turbulence in compressible mixing layer
}

\author{
FU Dexun (傅德葽) ${ }^{1}$, MA Yanwen (马延文) ${ }^{1}$ \& ZHANG Linbo (张林波) ${ }^{2}$ \\ 1. Laboratory of Nonlinear Mechanics, Institute of Mechanics, Chinese Academy of Sciences, Beijing 100080, China; \\ 2. State Key Laboratory of Scientific and Engineering Computing, Institute of Computational Mathematics and Spe- \\ cific/Engineering Computing, Chinese Academy of Sciences, Beijing 100080, China \\ Correspondence should be addressed to Fu Dexun (email: fud@ cc5 .imech.ac.cn)
}

Received June 18, 1999

\begin{abstract}
The three-dimensional compressible Navier-Stokes, equations sro approximated by a fifth order upwind compact and a sixth order symmetrisai scrnpatet ditiereicice relations combined with threestage Ronge-Kutta method. The computecl results ari presented for convective Mach number $M c=$ 0.8 and $R e=200$ with initial data which have gqual and opposite oblique waves. From the computed results we can see the variation: al cohereni structures with time integration and full process of instability, formation of $\Lambda$-virticies, joutie horseshoe vortices and mushroom structures. The large structures break into small and smsller vortex structures. Finally, the movement of small structure becomes dominant, and flow field turns into turbulence. It is noted that production of small vortex structures is combined with turning of symmetrical structures to unsymmetrical ones. It is shown in the present computation that the flow field turns into turbulence directly from initial instability and there is not vortex pairing in process of transition. It means that for large convective Mach number the transition mechanism for compressible mixing layer differs from that in incompressible mixing layer.
\end{abstract}

Keywords: compressible mixing layer, transition, turbulence, compact scheme.

Compressible mixing layer is a good model for studying turbulence. It also is a fundamental subject for understanding and solving many practical problems. For example, it is a basic research subject for improving the combustion efficiency in supersonic scramjet. Lots of research work have been done on incompressible mixing layers by experiments, theoretical analysis, and numerical simulations. Study on compressible mixing layer started just recently. In many investigations the numerical results were given only for the early stage of mixing, and many problems need to be solved.

In the early time Sandham and Reynolds ${ }^{[1]}$ investigated temporary developing compressible mixing layer for $M c=0.8$, and obtained process from initial instability to formation of $\wedge$-vortices. They supposed that vortex pairing will follow the formation of $\Lambda$-vortices. Later Luo and Sandham ${ }^{[2]}$ showed that the mechanism of transition in compressible mixing layer differs from that in incompressible mixing layer. They noted that the transition to turbulence starts directly from initial instability, and there is not second instability and vortex pairing. In their computation it is supposed that in process of transition the coherent structures for $M c=0.8$ are symmetrical in $z$ direction. This restriction is too strong for simulation of turbulence. Many problems remain to be solved in future, such as the mechanism of transition, effect of compressibility, and the reason for decreasing growth rate with the increasing convective Mach number. 
In the present paper the high order accurate method developed in ref. [3] is used to approximate the three-dimensional compressible Navier-Stokes equations and simulate the temporary developing mixing layer with convective Mach number $M c=0.8$ and $R e=200$. The convective Mach number is defined by $M c=\left(u_{1}-u_{2}\right) /\left(c_{1}+c_{2}\right)$, where $u_{1}, u_{2}$ and $c_{1}, c_{2}$ are upper and lower incoming velocity and sound speed respectively. Full process from initial instability to transition and to turbulence is obtained. From the computed results it can be seen that the coherent

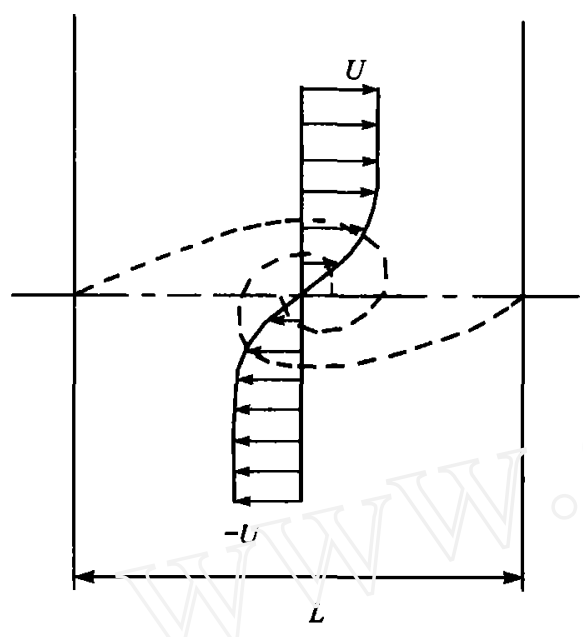

Fig. 1. Schematic diagram. structures are turning from early stage symmetry to later stage unsymmetry. The flow structures differ from that in incompressible mixing layer. With initial conditions used in the present paper the transition to turbuience starts directly froin init:a! instaóility without vortex pairing.

i Governing equations and numerical methods

The schematic diagram of temporary developing mixing layer is shown in fig. 1. In order to get fine mesh grid near the central part of domain a coordinate transformation is used in the normal direction.

For complete gas the dimensionless Navier-Stokes equations after coordinate transformation in vector form are as follows:

$$
\frac{\partial U}{\partial t}+\frac{\partial f_{1}}{\partial x}+\frac{\partial f_{2}}{\partial y}+\frac{\partial f_{3}}{\partial z}=\frac{\partial F_{1}}{\partial x}+\frac{\partial F_{2}}{\partial y}+\frac{\partial F_{3}}{\partial z},
$$

where

$$
\begin{gathered}
U=\frac{1}{N}[\rho, \rho u, \rho v, \rho w, E, \rho g]^{\mathrm{T}}, \\
f_{1}=\frac{1}{N}\left[\rho u, \rho u^{2}+p, \rho u v, \rho u w, u(E+p), \rho g u\right]^{\mathrm{T}}, \\
f_{2}=\left[\rho v, \rho u v, \rho v^{2}+p, \rho v w, v(E+p), \rho g v\right]^{\mathrm{T}}, \\
f_{3}=\frac{1}{N}\left[\rho w, \rho w u, \rho w v, \rho w^{2}+p, w(E+p), \rho g w\right]^{\mathrm{T}}, \\
E=\rho\left[C v T+\left(u^{2}+v^{2}+w^{2}\right) / 2\right],
\end{gathered}
$$

where $\rho, p, u, v, w$ and $T$ are density, pressure, three velocity components and temperature. They are normalized by $\rho_{\infty}, \rho_{\infty} u_{\infty}^{2}, u_{\infty}, u_{\infty}, u_{\infty}$ and $T_{\infty}$ respectively. The subscript $\infty$ shows that the flow parameters are in free incoming conditions. $F_{1}, F_{2}, F_{3}$ on right hand side in equations are the viscous terms. In dimensionless form we have

$$
p=\rho T / r M_{\infty}^{2}, C v=1 /\left[r(r-1) M_{\infty}^{2}\right],
$$

where $r$ is the ratio of specific heats, and $M_{\infty}$ is the incoming Mach number. The viscosity coefficient $\mu$ is computed by using the Sutherlands relation. The Reynolds number $R e$ is defined by $R e$ $=\frac{\rho_{\infty} u_{\infty} \delta_{w o}}{\mu}$, where $\delta_{w o}$ is the initial vorticity thickness. The quantity $\rho g$ in eq. (1) can be re- 
garded as the concentration per unit volume of a trace species.

The method used in the paper basically is the same as in ref. [3], but the group velocity control is not used because there are no shocks for the case $M c=0.8$. The fifth order accurate upwind compact difference relation developed by the author ${ }^{[4]}$ is used to approximate the convection terms, and a sixth order symmetrical compact difference relation ${ }^{[5]}$ is used to approximate the viscous terms, and a three-stage Rung-Kutta method is used in advance of time. In $x$ and $z$ directions periodical boundary conditions are used, and nonreflection condition is used in $y$ direction.

For simplicity consider the following model equation:

$$
\frac{\partial U}{\partial t}+\frac{\partial f}{\partial y}=0
$$

After flux splitting we have

$$
\frac{\partial U}{\partial t}+\frac{\partial f^{+}}{\partial y}+\frac{\partial f^{-}}{\partial y}=0
$$

Suppose that the boundaries in $y$ direction are far $a^{\prime}$ ay from the perturbation region. The second order nonreflection boundary condition, for example at $j=i$, is as follows:

$$
j=1: F_{j}^{+}=0, F_{i} \quad \because \frac{1}{2}\left[3\left(f_{j+1}^{-}-f_{j}^{-}\right)-\left(f_{j+2}^{-}-f_{j+1}^{-}\right)\right] .
$$

At the point $r_{i} \in \mathrm{xt}$ to the boundery the following third order approximation is used:

$$
\begin{gathered}
j=2: \quad F_{j-1}^{+}=0, \\
\alpha F_{j}^{+}=a\left(f_{j+1}^{+}-f_{j}^{+}\right)+b\left(f_{j}^{+}-f_{j-1}^{+}\right),
\end{gathered}
$$

where $\alpha=\frac{4}{3}, a=\frac{1}{2}, b=\frac{5}{6}$, and $F_{j}^{ \pm} / \triangle y$ is approximation of $\partial f^{ \pm} / \partial y$. In the same way we can get approximation at upper boundary.

\section{Direct simulation of compressible mixing layer}

\subsection{Initial conditions}

The initial flow field consists of mean flow plus perturbations: $f=\bar{f}+f^{\prime}, f=\rho, u, v, w$, $T$. The streamwise mean velocity profile is a hyperbolic tangent function

$$
\begin{gathered}
\bar{u}=A[B+\tan h(\beta y)], \beta>0, \\
\bar{v}=\bar{W}=0, \\
A=\left(u_{1}-u_{2}\right) / 2, B=\left(u_{1}+u_{2}\right) / 2,
\end{gathered}
$$

where $u_{1}$ and $u_{2}$ are upper and lower incoming steamwise velocity components (fig. 1). In computation $u_{1}=-u_{2}=1, \beta=2, \operatorname{Pr}=1$. The initial mean temperature profile is

$$
\bar{T}=1+M_{1}^{2} \frac{r-1}{2}\left(1-\bar{u}^{2}\right),
$$

where $M_{1}$ is the upper incoming Mach number. The initial pressure distribution is assumed to be constant, and the density is obtained from the equation of state.

From linear stability analysis ${ }^{[6]}$ we know that the most unstable waves are three-dimensional oblique waves for compressible mixing layer for the case $M c=0.8$. The initial perturbation in the present paper is as follows:

$$
\begin{gathered}
f^{\prime}=a \operatorname{Re}\left\{\hat{f}(\alpha, \beta) \mathrm{e}^{\mathrm{i}(\alpha x+\beta z)}+\hat{f}(\alpha,-\beta) \mathrm{e}^{\mathrm{i}(\alpha x-\beta z)}\right\}, \\
f^{\prime}=p^{\prime}, u^{\prime}, v^{\prime}, w^{\prime}, T^{\prime} .
\end{gathered}
$$


In the above expression $\operatorname{Re}\left\{\hat{f}(\alpha, \beta) \mathrm{e}^{\mathrm{i}(\alpha x+\beta z)}+\hat{f}(\alpha,-\beta) \mathrm{e}^{\mathrm{i}(\alpha x-\beta z)}\right\}$ means the real part in the bracket, $\alpha, \beta$ are wave numbers in $x$ and $z$ directions respectively, $\hat{f}(\alpha, \beta)$ and $\hat{f}(\alpha,-\beta)$ are eigenfunctions corresponding to the most unstable waves obtained from linear theory from ref. [6]. In the present computation $M c=0.8, R e=\rho_{\infty} u_{\infty} \delta_{w a} / \mu=200, u_{\infty}=\left(u_{1}-u_{2}\right) / 2$,

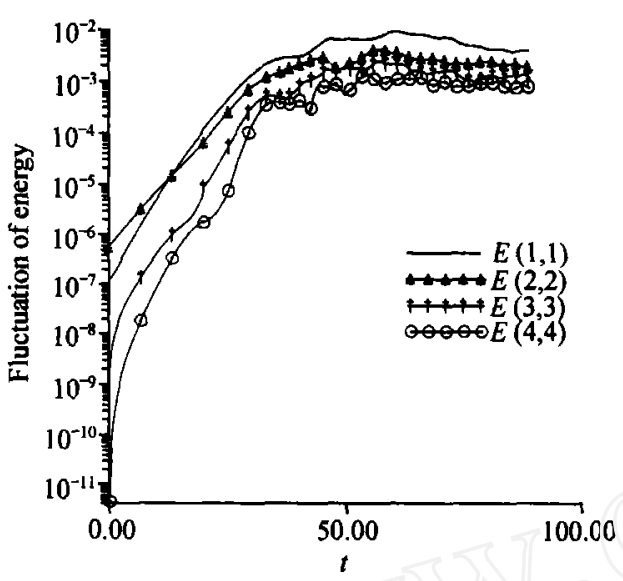

Fig. 2. Jaration of $E_{,}(\alpha, \beta)$ is. $t$. and $\delta_{w o}=\left(u_{1}-u_{2}\right) /\left|\frac{\mathrm{d} \bar{u}}{\mathrm{~d} y}\right|_{\max }$. For the case $M c=$ $0.8, \operatorname{Re}=200$, we have $\alpha=\beta=0.47$ from linear theory. The domain of computation is $0 \leqslant x \leqslant$ $2 \pi / \alpha ;-15 \leqslant y \leqslant 15 ; 0 \leqslant z \leqslant 2 \pi / \beta$.

\subsection{Results and analysis}

The mesh grid points irititially in $(x, y, z)$ directions are $64 \times 22 \mathrm{i} \times 54$. With iurning to small scale struetire dominance ine mesh grid is refined sequentially to $160 \times 245 \times 200$. For keeping accuracy a sixth order Lagrange interpolation is used in mesh refinement. In fig. 2 are given the two-dimensional averaged spectra of total fluctuation of energy,

which is deñwed as

$$
\begin{gathered}
E_{c}(\alpha, \beta)=\frac{1}{2 L_{y}} \int_{-L_{y}}^{L_{y}} \hat{E}_{c}^{\prime}(\alpha, \beta, y) \mathrm{d} y, \\
\hat{E}_{c}^{\prime}(\alpha, \beta, y)=\frac{1}{L_{x} L_{z}}\left|\int_{0}^{L_{x}} \int_{0}^{L_{s}} E_{c}^{\prime}(x, y, z) \mathrm{e}^{-2 \mathrm{i} \pi(\alpha x+\beta z)} \mathrm{d} x \mathrm{~d} z\right|, \\
E_{c}^{\prime}(x, y, z)=\frac{1}{2}\left(u^{\prime 2}+v^{\prime 2}+w^{\prime 2}\right),
\end{gathered}
$$

where $f=\tilde{f}+f^{\prime}$, and $\tilde{f}$ is the Fevre average and $\tilde{f}=\bar{\rho} f / \bar{\rho}$. The upper “-_ means Reynolds average. In fig. 2 the wave numbers are normalized by the basic wave number. From this figure we can see that in early time the perturbations have high wave numbers equal zero. With flow development the energy for small scale structures gets exited, and then the peturbation energy in a wide range of scales turns to quantity with almost equal order. It means that the small structures can get energy $\precsim$ from averaged large scale structures in order to keep their movement. This is indirect validation of the present computed results. In fig. 3 are given the maximum and minimum pressure $P_{\max }$ and $P_{\min }$ as a function, of time. From this figure we can see the process of transition. The minimal $P_{\min }$ in advance of time corresponds to different stage of flow development. In fig. 4 are given the surfaces of constant pressure

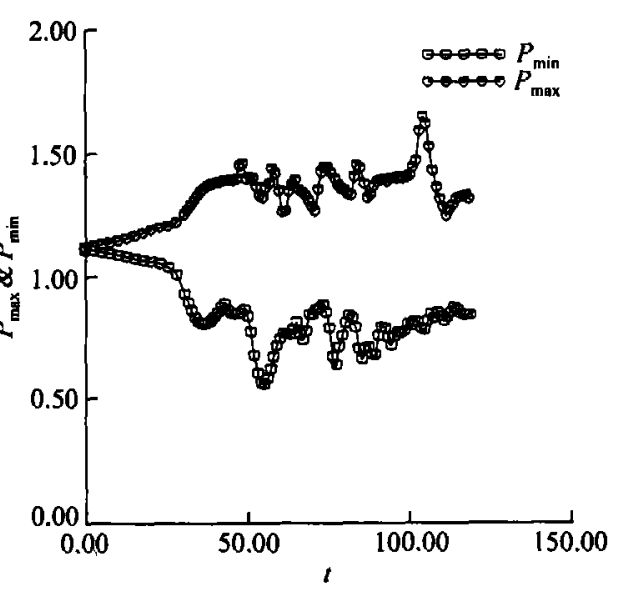

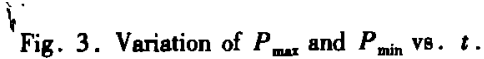



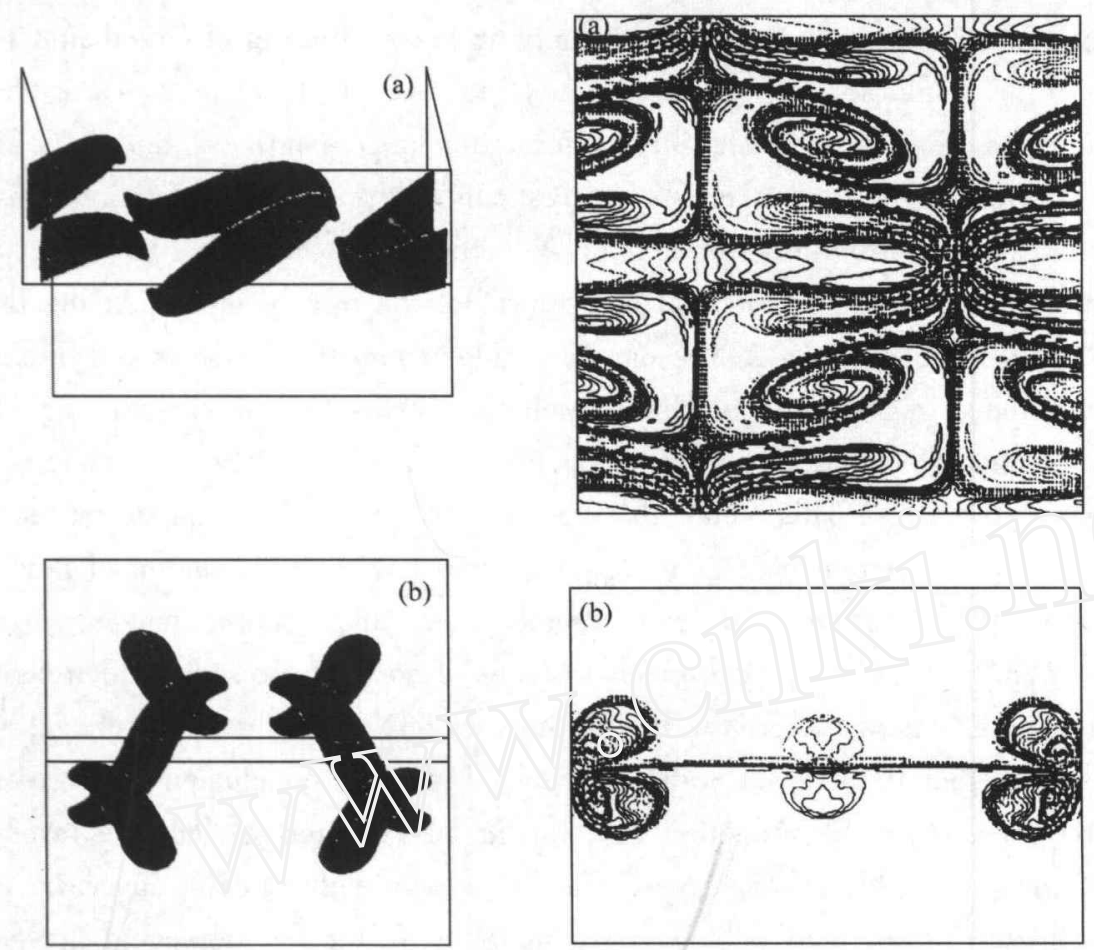

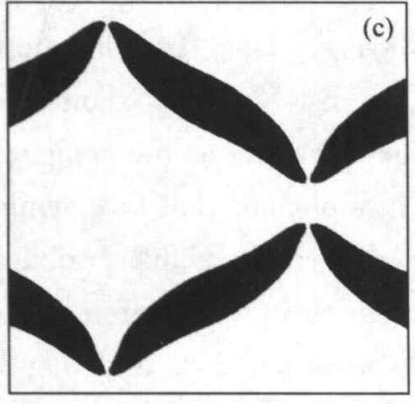

A

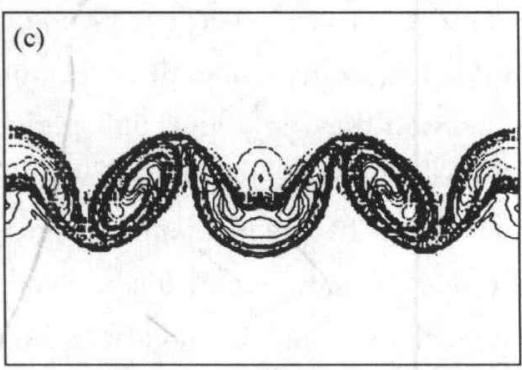

Fig. 4. A, Surface with constant pressure at $t=36.63$. (a) $3 D$ constant surface; (b) front view; (c) top riew. B, Contours of passive function at $t=36.63$. (a) Plane $y=0$; (b) Plane $x=\frac{1}{4} L_{x}$; (c) plane $x=\frac{1}{2} L_{\mathbf{v}}$.

(fig. $4 \mathrm{~A}(\mathrm{a}),(\mathrm{b})$ ) and mixture fraction field (fig.4B, the passive function $g$ ) at time $t=36.63$ which corresponds to the time when $P_{\min }(t)$ reaches its first minimal point. From the surfaces of constant pressure we can see formation of $\Lambda$-vortices. These structures much like the $\Lambda$-vortices for $\mathrm{H}$-type transition in the boundary layer. In the mixing layer formation of $\Lambda$-vortices is from primary instability, but in the boundary layer its formation is from instability for subharmonic ( secondary instability). These results agree well with those from refs. $[1,2]$ in which a spectral method combined with finite difference method is used ( see fig . $13 \mathrm{~b}$ in ref . [ 1 ] and fig . 2 in 
ref. [2]). The contours of passive function $g$ (fig. 4B(a)-(c)) characterize the property of mixture. Computed mushroom structure in the mixing layer which is observed first in experiment agrees well with the results in ref. [1] (see fig. 15 in ref. [1]). In ref. [1] are only given the results in the period from initial instability to formation of $\Lambda$-vortices, and it is supposed that vortex pairing follows the formation of $\Lambda$-vortices. In the present computation it is shown that with development of flow structures the ends of $\Lambda$-vortices start to incline in normal direction. In process of inclining we can see periodical fast growth of the mixing layer. At the time when the $\Lambda$-vortices starts to incline $(t \sim 42)$ we can see sudden growth of momentum thickness (fig. 5 (a)). The variation of maximum spanwise vorticity $\omega_{z}$ versus time is given in fig. 5(b). From the figure we see that $\omega_{z}$ is negative for $t<36$. Formation of $\Lambda$-vortices is combined by sudden growth of positive vorticities. This means that the nonlinear effect is an impriant factor for formation of large scale three-dimensional coherent structures. With continuation of flow development due to inclination of $\Lambda$-vortices and interaction between them double horseshoe structures are formed at $t=52.37$. In fig. 6(a) are givein surtaces of ocrstant pressure which show the coherent structures. These structures alree well with those in ref. [2] (fig. 2 in ref. [2]). The computed results tell us that there is no vortex pairing. The same conclusion is given in ref. [2] . This conclusion show:; that the transition mechanism for compressible mixing layer differs from mechanisn for uncompressible mixing layer. For the incompessible mixing layer the vortex pairing is a key factor in transition, and it also is an important factor for increasing the growth rate of mixing layer. For the compressible mixing layer there is no vortex pairing, and the mixing layer combined with inclination of $\Lambda$-vortices periodically grows very fast. The flow turns to turbulence directly from initial instability, and there is no secondary instabitity. It should be noted that in ref. [2] in $z$-direction they only took half period of basic hormonic as the computational domain, and another half period is supposed to be symmetrical. It is obvious that flow symmetry is not correct for turbulent flow. In the present computation in $z$-direction a full period is taken as the length of domain and periodic condition is used at boundaries in $z$-direction. In computation we see that the flow structures are symmetrical in $z$-direction for $t \leqslant 67$, and they agree well with symmetrical results in ref. [2]. With break of large scale structures and formation of small scale
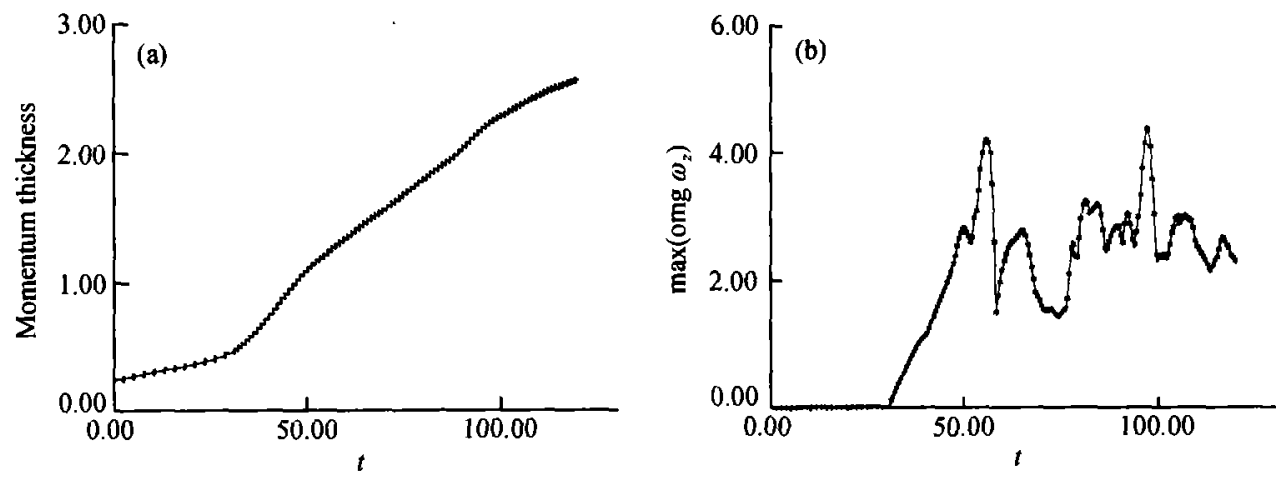

Fig. 5. (a) Variation of $\delta_{0} \mathrm{vs} . t$; (b) variation of $w_{z \max } \mathrm{vs} . t$ 

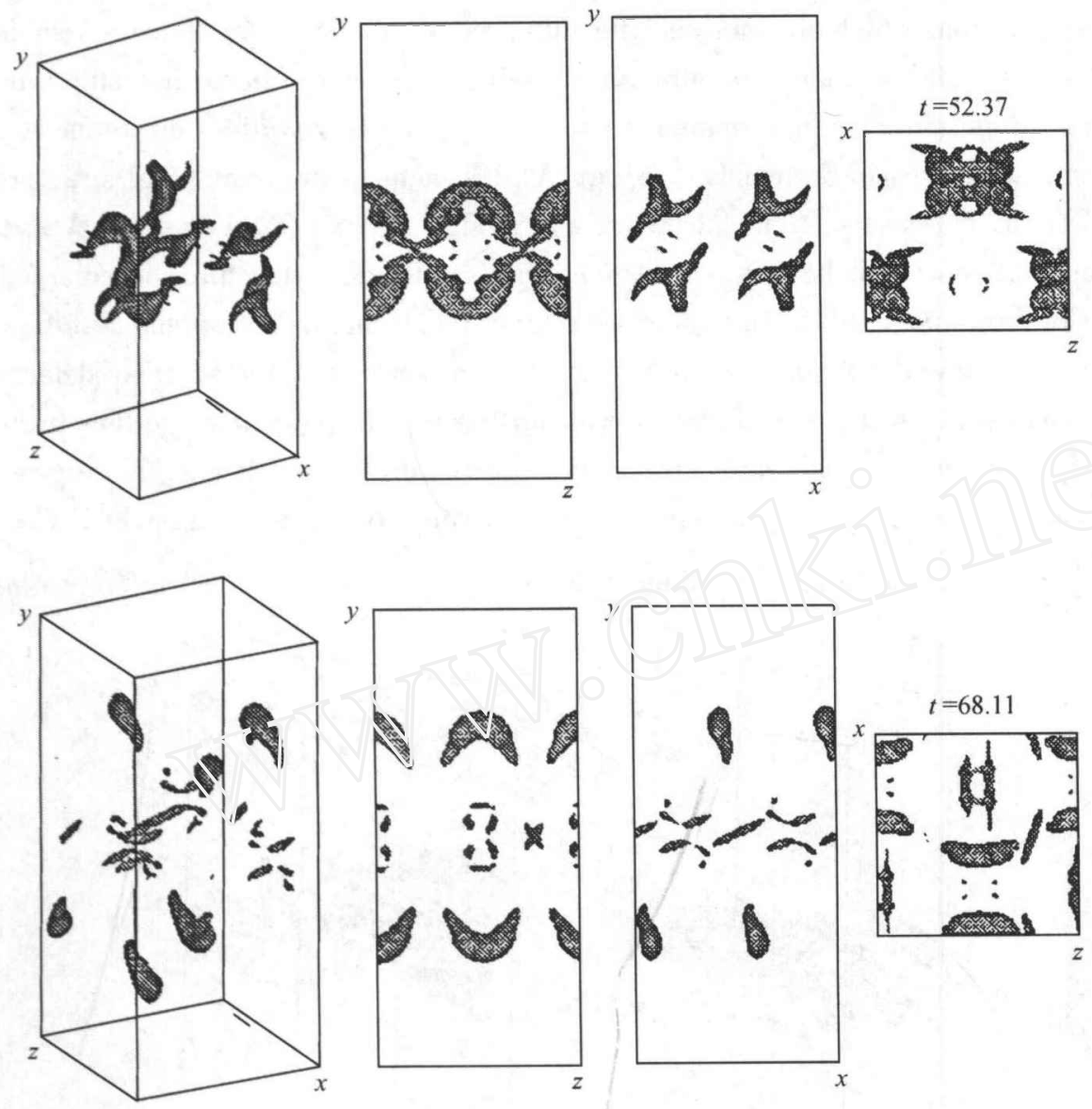

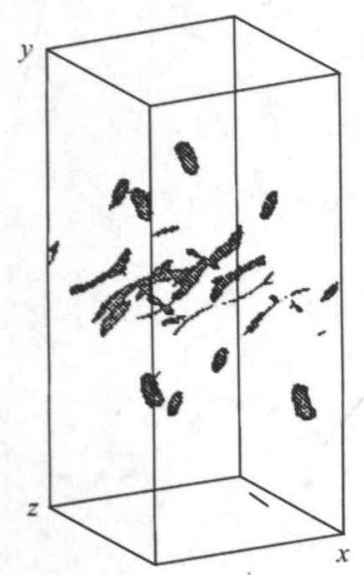

(a)

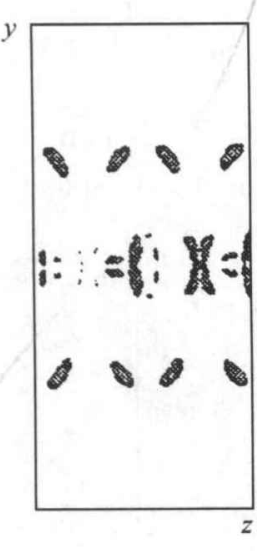

(b)

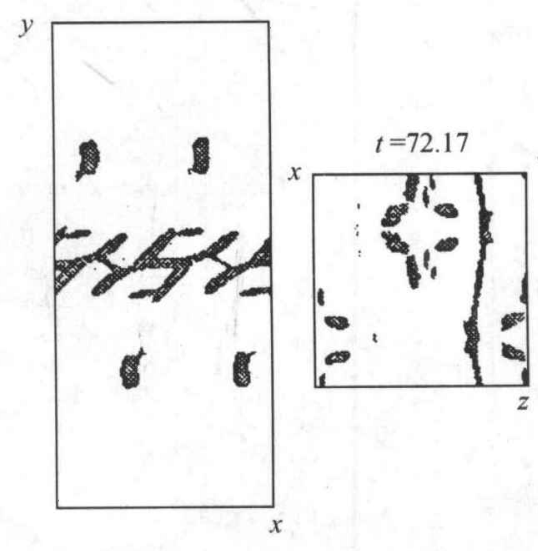

(c) (d)

Fig. 6. Surface of constant pressure. (a) 3D constant surface; (b) front view; (c) side view; (d) top view. 
structures unsymmetry in $z$-direction is obtained. In fig. 6 are given surfaces of constant pressure at different time, from which we can see the full process of flow development from large scale structures to smaller and smaller scale structures. With movement of horseshoe structures away along the normal direction after its formation $(t=52.37)$, the bow vortices are formed, and break into small scale structures in the central region. At this moment unsymmetrical structures are exhibited in $z$-direction (see fig. 6(b), 6(d) at $t=68.11$ ). At $t=72.17$ each bow vortex breaks into two small vortices, and the space in which exist small scale structures is enlarged. At this moment the flow structures still keep unsymmetry (fig. 6). From the computed results we can see the full process of flow development: stretching of large scale structures, their deformation and formation of small scale structures. Finally, with further flow development the flow curns into turbulence, and movement of small scale structures becomes dominant. In fis 7 are given the surfaces of constant spanwise vorticity $w_{z}$ and streamwise vorticity $\omega_{x}$ at $t=116.84$. The Reynolds stress $R_{12}=-\tilde{u^{\prime} v^{\prime}}$ and the turbulence Mach number $\boldsymbol{M}_{i}=\left(\tilde{u_{2}^{\prime} u_{i}^{\prime}}\right)^{3.5} / \tilde{c}$ at different time are giv-

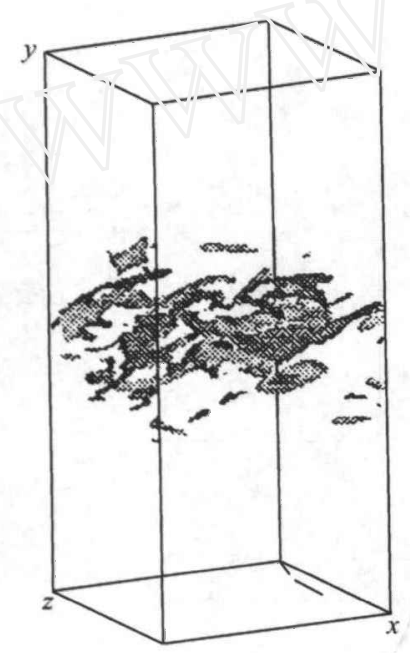

(a)

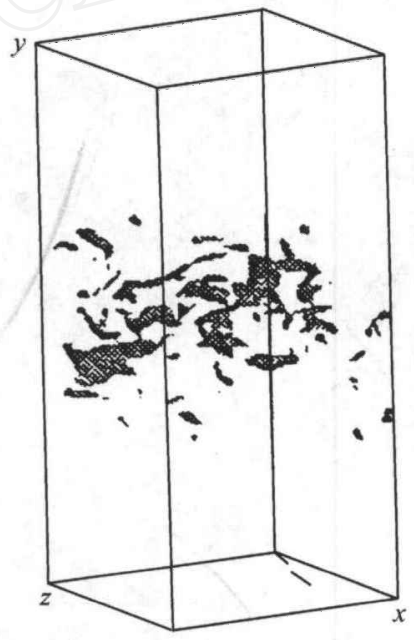

(b)

Fig. 7. Constant surface of pressure for $w_{3}(\mathrm{a})$ and $w_{3}$ (b) at $t=116.84$.
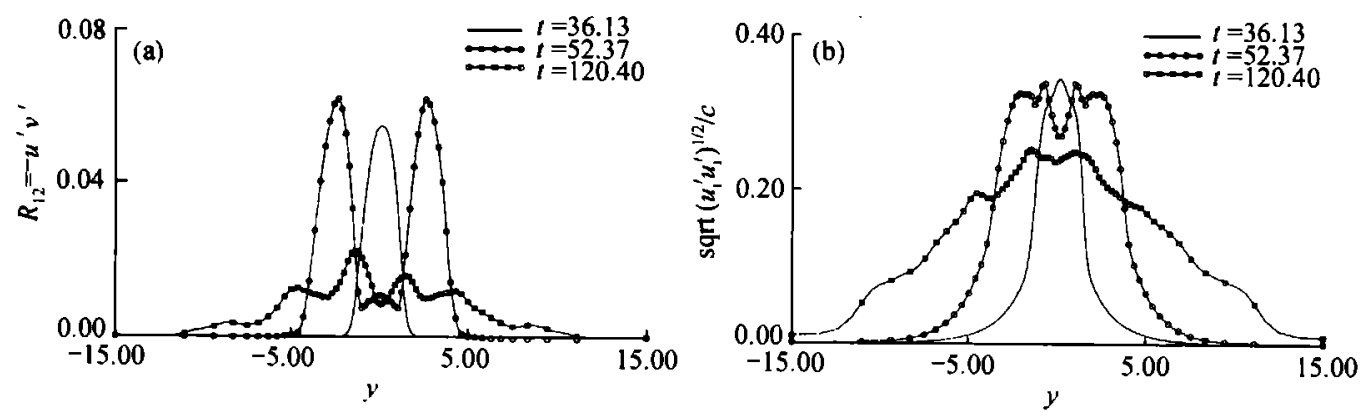

Fig. 8. Variation of $R_{12}$ and $M_{1}$ (b) in different time. 
en in fig. 8. Here upper" $\sim$ "means Favre average. Variation of $R_{12}$ and $M_{t}$ shows characteristics of flow structures in different stage of development. During the time of formation of $\Lambda$-vortices the stress $R_{12}$ as a function of $y$ has a single peak ; during the time of formation of horseshoe vortices $R_{12}$ has a pair of peaks, its production is due to shear stress caused by double horseshoe vortices. When the flow turns into turbulence $R_{12}$ has multipeak and becomes flatter. The turbulence Mach number has similar property. It should be noted that the Reynolds number in the present computation is lower. Although the small structures trend to isotropy, but full developed turbulence has not been reached.

\section{Conclusion}

The three-dimensional compressible mixing layer is simulated directly with a high order accurate method. The full process of flow development from initiai instak,ility, transition and early stage of turbulence are given. It is noted that during transition ploduction of small scale structures is combined with turning of flow to unsymmetry. These resilts show that for larger convective Mach number in compressible mixing layer the mechanism of iransition differs from that for incompressible mixing layer. The flow dievelopment with given initial conditions in the present paper is direct from initial instabiliiy to turivulence, and there are no paring and second instability.

Acknowledgements 'Th'3 work was supported by the National Natural Science Foundation of China (Grant No. 19872069) and State Key Project for Basic Research. The work was performed on computers of State Key Laboratory of Scientific and Engineering Computing, and Computer Network Information Center, Chinese Academy of Sciences.

\section{References}

1. Sandham, N. D., Reynolds, W. C., Three-dimensional simulation of large eddies in the compressible mixing layer, J. Fluid Mech., 1991, 244: 133 .

2. Luo, K. H., Sandham, N. D., Voke, P. R. eds., Direct and Large-Eddy Simulation I, Netherlands: Kluwer Academic Publishers, 1994, 335-346.

3. Fu Dexun, Ma Yanwen, Evolution of three-dimensional temporary developing mixing layer, Acta Mechanica Sinica (in Chinese), $1998,30(2): 129$.

4. Fu Dexun, Ma Yanwen, Computational Fluid Dynamics Review (eds. Hafez, M., Oshima, K.), New York: John Wiley \& Sons, 1995, 234-250.

5. Lee, S., Lele, S. K., Moin, P., Eddy shocklets in decaying compressible turbulence, Phys. Fluids A, 1991, $3: 657$.

6. Wang Qiang, Fu Dexun, Ma Yanwen, Numerical computation of stability for compressible mixing layer, Computational Physics, 1997, 6(4): 414 . 